\title{
ORIGINAL RESEARCH \\ Differences in Microstructural Alterations of the Hippocampus in Alzheimer Disease and Idiopathic Normal Pressure Hydrocephalus: A Diffusion Tensor Imaging Study
}

Y.J. Hong

B. Yoon

Y.S. Shim

A.H. Cho

S.C. Lim

K.J. Ahn

D.W. Yang

\begin{abstract}
BACKGROUND AND PURPOSE: DTI can be used to assess the degradation of WM tracts by measuring the FA and MD. Decreased FA/increased MD values in the AD hippocampus have been reported by many studies. In contrast, only a few studies reported on hydrocephalus by using the DTI technique. In elderly patients with dementia and with dilated ventricles, it is often difficult to differentiate iNPH from $A D$ with visual measurements on MR imaging. The aim of this study was to investigate the changes of microstructural integrity of the hippocampus in iNPH by using DTI and determining whether this method could be a new diagnostic tool to differentiate iNPH from AD.
\end{abstract}

MATERIALS AND METHODS: We recruited 43 participants ( 15 healthy controls, 15 patients with AD, and 13 patients with iNPH). The FA and MD values were measured by using the region-of-interest method in the hippocampal head, body, and tail on both sides. Clinical history, neurologic examination, and neuropsychological assessment were included.

RESULTS: The FA values were the lowest in the patients with AD, the patients with iNPH, and the healthy controls in this order. The MD values were the highest in the same order. These findings were consistent in the 3 subdivisions of the bilateral hippocampal regions. Hippocampal volume was not different between patients with iNPH and AD.

CoNCLUSIONS: The microstructural alterations of the hippocampus were more sensitive than the volumetric changes in $A D$ and $\mathrm{NPH}$. DTI analysis might be a useful tool for discriminating AD from iNPH.

\begin{abstract}
ABBREVIATIONS: $A D=$ Alzheimer disease; $A D C=$ apparent diffusion coefficient; $C D R=$ Clinical Dementia Rating Scale; CDR-SOB $=$ Clinical Dementia Rating Scale Sum of Boxes; $d f=$ degrees of freedom; $\mathrm{DTI}=$ diffusion tensor imaging; $\mathrm{FA}=$ fractional anisotropy; $\mathrm{HB}=$ hippocampal body; $\mathrm{HH}=$ hippocampal head; $\mathrm{HT}=$ hippocampal tail; iNPH = idiopathic normal pressure hydrocephalus; K-MMSE = Korean version of the Mini-Mental State Examination; Lt. = left; Rt. = right; $\mathrm{MD}=$ mean diffusivity; NPH = normal pressure hydrocephalus; SNR = signal-to-noise ratio; SPGR = spoiled gradient-recalled echo; $\mathrm{T} 2 \mathrm{WI}=\mathrm{T} 2$-weighted imaging; $\mathrm{WM}=$ white matter
\end{abstract}

D TI allows in vivo examination of the orientation and microstructural integrity of the WM by measuring FA and MD. ${ }^{1} \mathrm{FA}$, a quantitative measure of the degree of anisotropy, is reduced by changes in the tissue cytoarchitecture most likely due to subtle small-vessel alterations, demyelination of the axonal structures, and possibly gliosis. The MD, a quantitative measure of the mean motion of water, is increased by loss of neurons, axons, and dendrites. ${ }^{2}$

The FA and MD values in patients with $\mathrm{AD}$ have been measured in several studies. While many studies have consistently demonstrated reduced FA and increased MD values in the

\section{Received March 18, 2010; accepted after revision May 10.}

From the Departments of Neurology (Y.J.H., Y.S.S., A.H.C., S.C.L., D.W.Y.), and Radiology (K.J.A.), College of Medicine, The Catholic University of Korea, Seoul, Korea; and Department of Neurology (B.Y.), Konyang University College of Medicine, Daejeon, Korea.

This work was supported by a grant of the Korea Healthcare Technology R\&D Project, Ministry for Health, Welfare \& Family Affairs, Republic of Korea (A050079) and financial aid from the Catholic Medical Center Research Foundation, made in the program year 2009. Please address correspondence to Dong-Won Yang, MD, Department of Neurology, College of Medicine, The Catholic University of Korea, 505 Banpo-dong, Seocho-gu, Seoul, 137-701, Korea; e-mail:neuroman@catholic.ac.kr

\section{Indicates open access to non-subscribers at www.ajnr.org}

DOI 10.3174/ajnr.A2207 hippocampus and temporal, frontal, and parietal lobes of patients with $\mathrm{AD},{ }^{1-5}$ only a few studies have reported changes of the microstructural WM in patients with hydrocephalus by using DTI. ${ }^{6,7}$ Moreover, it is not known whether the hippocampus is susceptible to iNPH.

The hippocampus is a critical structure for learning and memory formation, and it is susceptible to a wide variety of neurologic diseases, including hypoxia-ischemia, epilepsy, schizophrenia, and AD. ${ }^{8}$ The hippocampus also exhibits characteristic pathologic features in patients with $\mathrm{AD}$, even in the very earliest stage of the disease. ${ }^{9}$

The syndrome of iNPH was first described by Hakim and Adams ${ }^{10}$; iNPH is characterized by the clinical triad of gait disturbance, dementia of the subcortical type, and urinary incontinence. It is different from $\mathrm{AD}$ in its pathologic mechanism and clinical findings. Making an early diagnosis and performing shunt surgery are mandatory because the results of shunt surgery in the late stage are often unsatisfactory. Greater improvements with shunt surgery would be expected in the early stage when the memory deficit is mild or absent. ${ }^{11}$ However, most iNPH cases are diagnosed among patients older than 60 years of age, who also have an increased risk for AD; and in elderly patients with dementia and dilated ventricles, it 
is often difficult to differentiate iNPH from $\mathrm{AD}$ with visual evaluation by using MR imaging.

We predicted that DTI is effective in differentiating iNPH and $\mathrm{AD}$ because there would be different patterns of microstructural WM change in patients with iNPH compared with patients with $\mathrm{AD}$. In this study, we measured the FA and MD values of the hippocampus in patients with $\mathrm{iNPH}, \mathrm{AD}$, and healthy controls to investigate the alteration of microstructural integrity and to assess the usefulness of DTI as a tool for differential diagnosis.

\section{Materials and Methods}

\section{Subjects}

Fifteen patients with AD, 13 patients with iNPH, and 15 healthy controls were enrolled from the Department of Neurology at St. Mary's Hospital of The Catholic University of Korea. Patients with AD fulfilled the National Institute of Neurologic and Communicative Disorders and Stroke and the Alzheimer Disease and Related Disorders Association criteria for probable AD. ${ }^{12}$ Patients with iNPH met the criteria for the diagnosis of probable iNPH according to the "Clinical Guidelines for Idiopathic Normal Pressure Hydrocephalus." 13 The criteria are the following: 1 ) older than 60 years of age; 2 ) having $\geq 1$ symptom of gait disturbance, dementia, or urinary incontinence; 3 ) ventricular dilation (Evan index $>0.3$ ) and a narrow CSF space in the superior convexity; 4) a CSF pressure lower than $20 \mathrm{~cm} \mathrm{H}_{2} \mathrm{O}$ with normal properties of the CSF cell counts and protein level; 5) no other diseases that may account for the symptoms; 6) no other previous illnesses that cause ventricular dilation; and 7) a positive spinal tap test result.

Subjects were excluded from enrollment if they were younger than 60 years of age or had a history of a disease that may cause cognitive impairment or major psychiatric disease.

Fifteen healthy volunteers were selected as controls. They had no cognitive impairment, their scores on the $\mathrm{K}_{-} \mathrm{MMSE}^{14}$ were above $-1.5 \mathrm{SDs}$, and their scores on the $\mathrm{CDR}^{15}$ were 0 . All of them underwent clinical assessment, neuropsychological examination, and a brain MR imaging that included DTI and SPGR images. The clinical assessments consisted of a neurologic and psychiatric history and neurologic examination. They were assessed for the 28 illnesses proposed by Christensen et $\mathrm{al}^{16}$ that may be associated with cognitive impairment. The Ethics Committee of The Catholic University Hospital of Korea approved this study, and all of the participants provided written informed consent.

\section{Image Acquisition and Processing}

We performed DTI by using a 1.5T MR imaging scanner (Signa Excite 11.0; GE Healthcare, Milwaukee, Wisconsin) with a single-shot spinecho echo-planar diffusion-weighted sequence, in addition to the full conventional MR imaging sequence. A series of axial diffusionweighted images with a diffusion-sensitizing gradient ( $\mathrm{b}$-value = $1000 \mathrm{~s} / \mathrm{mm}^{2}$ ) along 25 directions was obtained, as well as acquisition of the axial images without diffusion weighting $(b$-value $=0)$. The other diffusion parameters were as follows: $\mathrm{TR}=10,000 \mathrm{~ms}$, TE $=$ $83.3 \mathrm{~ms}$, matrix $=128 \times 128, \mathrm{FOV}=260 \times 260 \mathrm{~mm}, \mathrm{NEX}=1,33$ axial sections, and section thickness $=4 \mathrm{~mm}$ with no intersection gap. The SNR in the reference ( $b$-value $=0$ ) image was approximately $100 \%$, which helped to reduce the bias of the estimated magnetization transfer imaging metrics. ${ }^{17,18}$

We also performed SPGR MR imaging with the following param- eters: 128 contiguous images with a $1.0-\mathrm{mm}$ thickness acquired in the coronal plane, acquisition matrix $=256 \times 256, \mathrm{FOV}=210 \mathrm{~mm}$, $\mathrm{TR} / \mathrm{TE}=22 / 6 \mathrm{~ms}$, flip angle $=30^{\circ}, \mathrm{NEX}=1$, and an average mean imaging time of 12 minutes. We measured the volume of the bilateral hippocampus in the patients with $\mathrm{AD}$ and $\mathrm{iNPH}$ by using a regionof-interest analysis. The analysis software package, Analyze, Version 8.1 (Mayo Clinic Foundation, Rochester, Minnesota), was used for manual measurement of the volumes of the hippocampus of each side (www.analyzedirect.com). The DICOM files stored in the PACS were converted to Analyze files, and these were reformatted to cubic volume (3D) with resliced $0.97-\mathrm{mm}$ image thickness. To calculate the volume of each part, we multiplied areas by the section thickness and these values were summed. We used the anatomic boundaries of the hippocampus as proposed by Pruessner et al. ${ }^{19}$

The raw DTI data were processed with the freely shared software programs Volume-One (version 1.56) and dTV.II. These programs are available on-line at www.volume-one.org and www.ut-radiology. umin.jp/people/masutani/dTV.htm, respectively. FA and MD were measured in 3 regions of the hippocampus (head, body, and tail) on both sides. The regions of interest were 3D-based voxels of interest and were drawn manually in a $33-\mathrm{mm}^{3}$ spheric shape. All the regions of interest were placed on coronal sections by using the landmarks proposed by Bernasconi et al. ${ }^{20}$ The region of interest for the hippocampal head was placed on the upper lateral area of the hippocampus where the mamillary body was fully visible. The region of interest for the hippocampal body was placed on the upper lateral area of the hippocampus where the pons, third ventricle, and cerebral aqueduct were visible. The region of interest for the hippocampal tail was placed where it contacts the lower margin of the crus fornix (Fig 1). ${ }^{20} \mathrm{We}$ coregistered the T2WI of the subjects by using the "Add computational channels" tool using the dTV.II program and found the correct landmarks by comparing the various images with various planes. The mean FA and MD values in the 3 sites of the hippocampus were calculated by taking measurements 3 times in random order by the same neurologist (L.S.C.), who was blinded to the patients' diagnosis. The intraclass correlation coefficients of each measurement were from 0.763 to 0.927 for this rater.

\section{Statistical Analysis}

The nonparametric Kruskall-Wallis test and the Mann-Whitney $U$ test were used for comparison of age, the K-MMSE score, the CDR/ CDR-SOB scores, the mean FA values, and the MD values among the 3 groups. $\chi^{2}$ tests were used for assessing the distribution of sex. The volume of the hippocampus on each side was compared between the $\mathrm{AD}$ and iNPH groups by using the nonparametric Mann-Whitney $U$ test. The statistical analysis was performed with the Statistical Package for the Social Sciences, Version 16.0 (SPSS, Chicago, Illinois). All the statistical tests were performed at the $5 \%$ confidence level of significance.

\section{Results}

The mean age of all subjects was $71.26 \pm 6.67$ years of age. The mean educational level was $8.74 \pm 5.40$ years. There were no significant differences in age, sex distribution, or education levels among the 3 groups. The K-MMSE (AD, $19.73 \pm 2.96$; $\mathrm{NPH}, 21.85 \pm 4.38)$ and the CDR scores $(\mathrm{AD}, 0.97 \pm 0.48$; $\mathrm{NPH}, 0.62 \pm 0.30)$ were not significantly different between the $\mathrm{iNPH}$ and $\mathrm{AD}$ groups. However, the CDR-SOB scores (AD, $4.9 \pm 2.96$; NPH, $2.58 \pm 2.29)$ were higher $(P=.025)$ in the patients with $\mathrm{AD}$ (Table 1). 

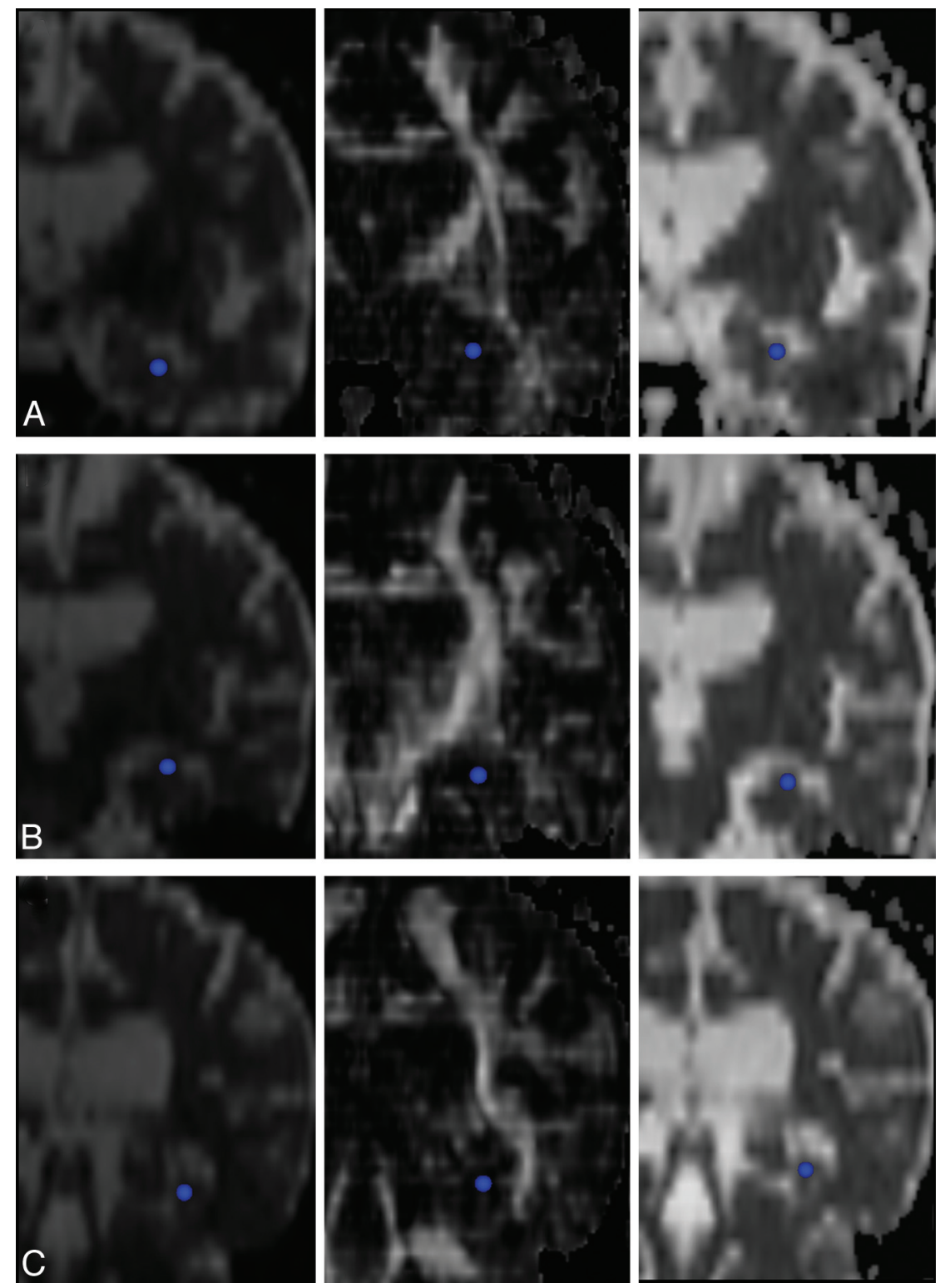

Fig 1. Regions of interest (blue) in 3 subdivisions of the hippocampus (head, $A$; body, $B$; and tail, $C$ ) on different images (coregistered T2WI, FA map, and ADC map; left-to-right direction).

\begin{tabular}{|c|c|c|c|c|}
\hline & Healthy (a) & $A D(b)$ & iNPH (c) & Post Hoc $(5 \%)$ \\
\hline Age (yr) & $70.07 \pm 3.83$ & $71.67 \pm 9.36$ & $72.15 \pm 5.79$ & $a=b=c$ \\
\hline Sex & $46.7 \%$ (male) & $46.7 \%$ (male) & $46.2 \%$ (male) & \\
\hline K-MMSE & $28.2 \pm 1.21$ & $19.73 \pm 2.96$ & $21.85 \pm 4.38$ & $a>b, c$ \\
\hline Education & $9.89 \pm 4.69$ & $8.67 \pm 5.64$ & $7.50 \pm 5.99$ & $a=b=c$ \\
\hline CDR score & 0 & $0.97 \pm 0.48$ & $0.62 \pm 0.30$ & $a<b, c$ \\
\hline CDR-SOB & 0 & $4.90 \pm 2.96$ & $2.58 \pm 2.29$ & $b>c>a$ \\
\hline
\end{tabular}

${ }^{a}$ Values are expressed as mean \pm SD. Age, K-MMSE, education levels, CDR score, and CDR-SOB scores were analyzed using the nonparametric Kruskal-Wallis test. Sex distribution was analyzed using the $\chi^{2}$ test. K-MMSE and CDR scores between AD and iNPH were not significantly different $(P>.05$ in the nonparametric Mann-Whitney $U$ test).

Because there were no significant differences between the right and left hippocampal sizes (left/right: $20.535 \pm 2.989$ $\mathrm{mm}^{3} / 21.119 \pm 2.815 \mathrm{~mm}^{3}$ in the AD group; $22.334 \pm 3.644$ $\mathrm{mm}^{3} / 22.899 \pm 3.600 \mathrm{~mm}^{3}$ in the iNPH group, $\left.P>.05\right)$, the mean values of both hippocampi were calculated. To correct for size variation of brains, we divided the hippocampal volume of each subject by the total intracranial volume. The hippocampal-volume ratios in both groups were not significantly different $(P>.05)$ (Table 2).
The mean FA values were the highest in the healthy controls, the iNPH group, and the AD group in this order $(P<$ $.001)$. The mean MD values were the lowest in the same order $(P<.001)$. The hippocampal tail showed the highest FA values and the lowest MD values among the 3 regions (head, body, and tail) of the hippocampus (Table 3 and Figs 2 and 3 ).

The healthy controls and the patients with iNPH showed no difference in the mean FA (left/right: $0.14 \pm 0.01 / 0.15 \pm$ 
Table 2: Mean hippocampal volumes and hippocampal volume ratios in AD and $\mathrm{NPH}^{\mathrm{a}}$

\begin{tabular}{lrrr}
\hline Volume & AD & iNPH & \\
\hline Hippocampal $\left(\mathrm{mm}^{3}\right)$ & $20.827 \pm 2.797$ & $22.617 \pm 3.617$ & $>.05$ \\
Hippocampal/total intracranial (\%) & $1.497 \pm 0.253$ & $1.603 \pm 0.244$ & $>.05$ \\
\hline
\end{tabular}

a Values are expressed as mean \pm SD. Data were analyzed by using the nonparametric Mann-Whitney $U$ test. Hippocampal volume ratio was not significantly different between the 2 groups.

\begin{tabular}{|c|c|c|c|c|c|c|c|}
\hline & Healthy (group 1) & $A D$ (group 2) & NPH (group 3) & $F$ Value & $d f$ & $P$ value & Post Hoc $(5 \%$ \\
\hline \multicolumn{8}{|l|}{ Mean FA } \\
\hline Lt. HH & $0.136 \pm 0.009$ & $0.082 \pm 0.007$ & $0.106 \pm 0.011$ & 131.88 & 2.40 & 0.000 & $1>3>2$ \\
\hline Lt. HB & $0.134 \pm 0.013$ & $0.086 \pm 0.011$ & $0.104 \pm 0.010$ & 68.59 & 2.40 & 0.000 & $1>3>2$ \\
\hline Lt. HT & $0.148 \pm 0.008$ & $0.093 \pm 0.008$ & $0.112 \pm 0.011$ & 148.05 & 2.40 & 0.000 & $1>3>2$ \\
\hline Rt. HH & $0.136 \pm 0.012$ & $0.094 \pm 0.011$ & $0.108 \pm 0.014$ & 44.43 & 2.40 & 0.000 & $1>3>2$ \\
\hline Rt. HB & $0.146 \pm 0.019$ & $0.092 \pm 0.012$ & $0.102 \pm 0.008$ & 65.14 & 2.40 & 0.000 & $1>3>2$ \\
\hline Rt. HT & $0.155 \pm 0.018$ & $0.102 \pm 0.009$ & $0.115 \pm 0.011$ & 61.90 & 2.40 & 0.000 & $1>3>2$ \\
\hline \multicolumn{8}{|l|}{ Mean MD } \\
\hline Lt. HH & $0.844 \pm 0.044$ & $1.399 \pm 0.186$ & $1.085 \pm 0.082$ & 78.21 & 2.40 & 0.000 & $2>3>1$ \\
\hline Lt. HB & $0.816 \pm 0.063$ & $1.283 \pm 0.240$ & $1.007 \pm 0.069$ & 35.99 & 2.40 & 0.000 & $2>3>1$ \\
\hline Lt. HT & $0.800 \pm 0.055$ & $1.230 \pm 0.137$ & $0.975 \pm 0.084$ & 71.91 & 2.40 & 0.000 & $2>3>1$ \\
\hline Rt. $\mathrm{HH}$ & $0.816 \pm 0.080$ & $1.249 \pm 0.171$ & $1.086 \pm 0.113$ & 43.96 & 2.40 & 0.000 & $2>3>1$ \\
\hline Rt. HB & $0.780 \pm 0.043$ & $1.151 \pm 0.181$ & $1.005 \pm 0.093$ & 35.70 & 2.40 & 0.000 & $2>3>1$ \\
\hline Rt. HT & $0.781 \pm 0.087$ & $1.152 \pm 0.204$ & $0.998 \pm 0.105$ & 25.28 & 2.40 & 0.000 & $2>3>1$ \\
\hline
\end{tabular}

${ }^{a}$ Data are expressed as mean FA and MD values \pm SD. Data were analyzed using the nonparametric Kruskal-Wallis test $(P$ value) and analysis of variance $(F$ value, $d f)$ with post hoc analysis (Bonferroni/Dunnett T3 methods according to homogeneity of variances).

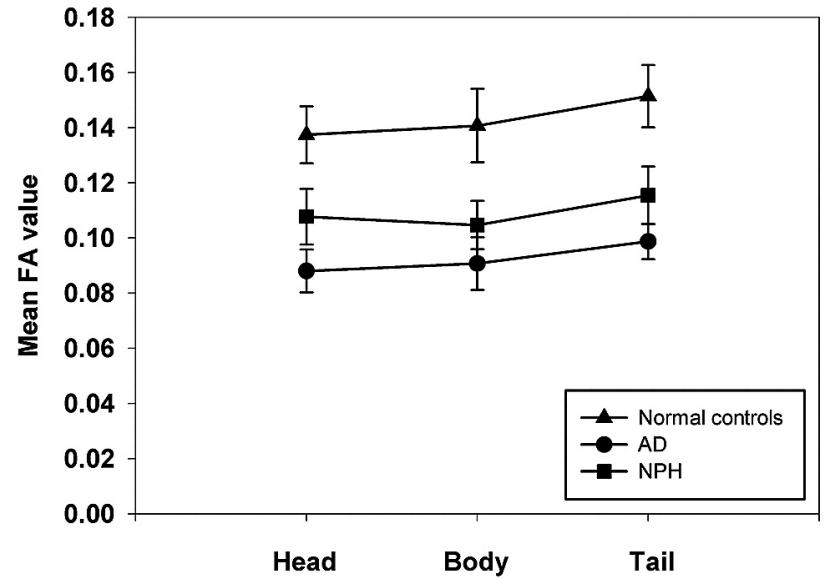

Fig 2. Mean FA values of both hippocampi in each subdivision of the hippocampus. Mean FA values are the lowest in $A D$ and the highest in healthy controls. This pattern is consistent in 3 different subdivisions of the hippocampus $(P<.001)$

0.01 in the healthy controls; $0.11 \pm 0.00 / 0.11 \pm 0.01$ in the patients with iNPH) and MD values (left/right: $0.82 \pm 0.04 /$ $0.79 \pm 0.04$ in the healthy controls; $1.02 \pm 0.05 / 1.03 \pm 0.06$ in the patients with iNPH) between the left and right hippocampi $(P>.05)$. Compared with that of the right side, the left hippocampus showed lower FA values (left/right: $0.09 \pm 0.01 /$ $0.10 \pm 0.01, P<.05)$ and higher MD values $(1.30 \pm 0.09 /$ $1.18 \pm 0.12, P<.05)$ in the patients with $\mathrm{AD}$.

\section{Discussion}

The validity of DTI as a sensitive tool for detecting subtle microstructural changes has been repeatedly demonstrated. ${ }^{4,21,22}$ A recent study also reported satisfactory intra- and interobserver precision of the FA and MD measures. ${ }^{23}$ We found that DTI would be an effective tool for differentiating patients with $\mathrm{AD}$ and $\mathrm{iNPH}$, who have little volume difference of the hippocampus on conventional structural MR imaging. Addition-

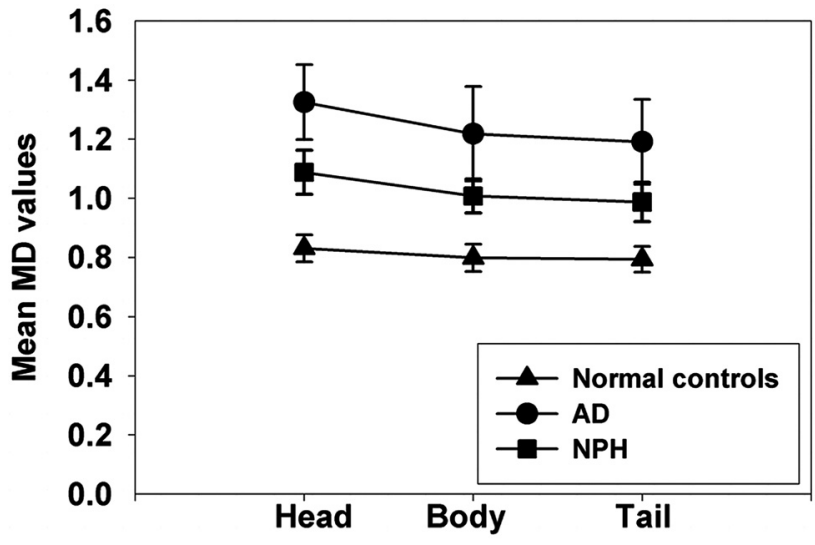

Fig 3. Mean MD values of both hippocampi in each subdivision of the hippocampus. Mean $\mathrm{MD}$ values are the highest in $\mathrm{AD}$ and the lowest in healthy controls $(P<.001)$. This pattern is consistent in 3 different subdivisions of the hippocampus.

ally, we confirmed that the microstructural change of the hippocampus has selective vulnerability.

Our results showed that patients with AD had significantly lower FA values and higher MD values in all 3 regions of the hippocampus compared with healthy controls and patients with NPH. The patients with iNPH showed lower FA values and higher MD values than the healthy controls. The present results could be interpreted as follows: First, the degree of anisotropy and water diffusivity in the hippocampus was changed the most in patients with $\mathrm{AD}$. Those results may demonstrate that WM as well as gray matter is damaged in patients with $\mathrm{AD}$. The WM has a close anatomic and functional connection to the overlying gray matter because it consists of either axons of the neurons in the overlying gray matter or axons reaching from other gray matter that have a synaptic connection with the neurons. ${ }^{24}$ The hippocampus is a heterogeneous structure, which is intermixed with gray matter and WM. The $\mathrm{AD}$ pathology, neurofibrillary tangles and amyloid plaque, de- 
posits in the hippocampal gray matter even in the early stage of the disease process. Hippocampal WM is also damaged, maybe due to wallerian degeneration of the WM tract connected to the gray matter.

Several previous studies have demonstrated decreased FA and/or increased MD values in the hippocampus with $\mathrm{AD}^{24,25}$ and mild cognitive impairment. ${ }^{4,24-27}$ Our study also pertains to the importance of differences in fiber integrity in the hippocampus among the healthy controls and patients with AD. Moreover, these data extend these findings to individuals with dementia of a different pathophysiology — that is, patients with iNPH.

There have been no previous studies that have compared FA and MD values among healthy controls, patients with iNPH, and patients with AD by using DTI analysis. The strength of this finding is emphasized by the fact that the hippocampal FA and MD values in patients with $\mathrm{AD}$ were clearly different from those of patients with iNPH.

Second, the patients with iNPH also showed decreased FA values and increased MD values compared with the healthy controls. The classic symptom triad of iNPH (gait disturbance, urinary incontinence, and cognitive decline) comes from the compression of the subcortical WM tracts around the lateral ventricles. The hippocampus is also compressed by the dilated temporal horn of the lateral ventricle. With prolonged exposure to increased intracranial pressure, hippocampal microstructures could be damaged due to various etiologies. Mechanical compression may change the metabolism of the monoamine neurotransmitters in the hippocampus. ${ }^{28}$ Altered metabolism of the hippocampus in patients with iNPH may also result in ischemic injury to some extent. ${ }^{9}$ Altered metabolism and ischemic injury could cause structural changes. However, these changes would be less severe than those of a primary degenerative dementia like AD. The 2 groups showed no significant difference in their hippocampal volumes; on the other hand, FA and MD values of the 2 groups were significantly different.

These results indicate that the mean FA and MD values by using DTI are more sensitive diagnostic parameters for differentiating $\mathrm{AD}$ from iNPH than manual volumetry. Clinically, differentiating iNPH from AD may be sometimes difficult in the elderly. Using DTI, we could detect the microstructural damage in patients with $\mathrm{iNPH}$ and $\mathrm{AD}$ and get a clue for differentiating the 2 disease conditions even in their early stages.

We also compared the mean FA and MD results in the 3 subdivisions of the hippocampus. The mean FA and MD values were different in each region of the hippocampus: head, body, and tail. The mean FA values were the highest in the tail and lowest in the head. The mean MD values were the lowest in the tail and highest in the head even in healthy controls. This anterior-to-posterior gradient could be explained by the heterogeneous tissue composition in the hippocampal subregions. ${ }^{29}$ Previous studies by using DTI proved the different cytoarchitectural features in the human and rat hippocampus. ${ }^{8,30}$ The water diffusion and the FA in the laminae of the hippocampus were found to be significantly different.

Our study had several limitations. First, the sample size was too small, so the results need to be interpreted with caution. However, we carefully matched the basic demographic data of the 3 groups, as described in Table 1. The AD and iNPH groups were also matched for the K-MMSE and CDR scores except the CDR-SOB scores. Our findings showed clearly different DTI parameters among the 3 groups, even though the sample size was small. The other limitation was the absence of pathologic confirmation. There are cases with concomitant pathologic abnormalities - for example, AD pathology in the iNPH group or vice versa that might have contaminated the results. ${ }^{9}$ Amyloid neuroimaging such as Pittsburgh Compound- ${ }^{31}$ or $2-\left(1-\left\{6-\left[\left(2-{ }^{18} \mathrm{~F}-\right.\right.\right.\right.$ fluoroethyl $)($ methyl $)$ amino $]-$ 2-naphthyl\}ethylidene) malononitrile (FDDNP) positronemission tomography ${ }^{32}$ will be useful to evaluate the concomitant pathologic status of patients with iNPH. We adopted the criteria of "probable iNPH" by using a clinical guideline to exclude the ambiguous iNPH cases.

Finally, there was a possibility that other gray matter structures or CSF components near the hippocampus could have been analyzed together. The low resolution of DTI results in a high chance of CSF partial volume contamination. The hippocampal regions of interest were carefully placed to exclude the perihippocampal CSF spaces and other gray matter structures by comparing the various images (FA map, color-coded map, ADC map, diffusion-weighted imaging map, and T2WI) with various planes (axial, coronal, and sagittal) of the axis. Moreover, we used relatively small-sized regions of interest, and we measured the mean FA and MD values 3 times at each subdivision of the hippocampus.

\section{Conclusions}

DTI is becoming increasingly important for investigating the microstructural properties of brain tissues. DTI analysis might be a useful tool for evaluating the microstructure in specific regions of the brain for making the differential diagnosis of patients with dementia. Further studies including larger samples of patients with more comprehensive neuropsychological tests and molecular neuroimaging will be needed to confirm the validity and reliability of measuring the FA and MD values in the hippocampus to differentiate iNPH from AD.

\section{References}

1. Mielke MM, Kozauer NA, Chan KC, et al. Regionally-specific diffusion tensor imaging in mild cognitive impairment and Alzheimer's disease. Neuroimage 2009;46:47-55

2. Rose SE, Janke AL, Chalk JB. Gray and white matter changes in Alzheimer's disease: a diffusion tensor imaging study. J Magn Reson Imaging 2008;27:20-26

3. Medina D, DeToledo-Morrell L, Urresta F, et al. White matter changes in mild cognitive impairment and AD: a diffusion tensor imaging study. Neurobiol Aging 2006;27:663-72

4. Fellgiebel A, Wille P, Mller MJ, et al. Ultrastructural hippocampal and white matter alterations in mild cognitive impairment: a diffusion tensor imaging study. Dement Geriatr Cogn Disord 2004;18:101-08

5. Parente DB, Gasparetto EL, da Cruz LC, et al. Potential role of diffusion tensor MRI in the differential diagnosis of mild cognitive impairment and Alzheimer's disease. AJR Am J Roentgenol 2008;190:1369-74

6. Yuan W, Mangano FT, Air EL, et al. Anisotropic diffusion properties in infants with hydrocephalus: a diffusion tensor imaging study. AJNR Am J Neuroradiol 2009;30:1792-98

7. Assaf Y, Ben-Sira L, Constantini S, et al. Diffusion tensor imaging in hydrocephalus: initial experience. AJNR Am J Neuroradiol 2006;27:1717-24

8. Shepherd TM, Ozarslan E, Yachnis AT, et al. Diffusion tensor microscopy indicates the cytoarchitectural basis for diffusion anisotropy in the human hippocampus. AJNR Am J Neuroradiol 2007;28:958-64

9. Savolainen S, Laakso MP, Paljrvi L, et al. MR imaging of the hippocampus in normal pressure hydrocephalus: correlations with cortical Alzheimer's disease confirmed by pathologic analysis. AJNR Am J Neuroradiol 2000;21: 409-14

10. Hakim S, Adams RD. The special clinical problem of symptomatic hydroceph- 
alus with normal cerebrospinal fluid pressure: observations on cerebrospinal fluid hydrodynamics. J Neurol Sci 1965;2:307-27

11. Meier U, Miethke C. Predictors of outcome in patients with normal-pressure hydrocephalus. J Clin Neurosci 2003;10:453-59

12. McKhann G, Drachman D, Folstein M, et al. Clinical diagnosis of Alzheimer's disease: report of the NINCDS-ADRDA Work Group under the auspices of Department of Health and Human Services Task Force on Alzheimer's Disease. Neurology 1984;34:939-44

13. Ishikawa M. Clinical guidelines for idiopathic normal pressure hydrocephalus. Neurol Med Chir (Tokyo) 2004;44:222-23

14. Kang YW, Na DL, Hahn SH. A validity study on the Korean Mini-Mental State Examination (K-MMSE) in dementia patients. J Korean Neurol Assoc 1997;15:300-08

15. Morris JC. The Clinical Dementia Rating (CDR): current version and scoring rules. Neurology 1993;43:2412-14

16. Christensen KJ, Moye J, Armson RR, et al. Health screening and random recruitment for cognitive aging research. Psychol Aging 1992;7:204-08

17. Basser PJ, Pierpaoli C. Microstructural and physiological features of tissues elucidated by quantitative-diffusion-tensor MRI. J Magn Reson B 1996;111: 209-19

18. Hasan KM, Narayana PA. Computation of the fractional anisotropy and mean diffusivity maps without tensor decoding and diagonalization: theoretical analysis and validation. Magn Reson Med 2003;50:589-98

19. Pruessner JC, Li LM, Serles W, et al. Volumetry of hippocampus and amygdala with high-resolution MRI and three-dimensional analysis software: minimizing the discrepancies between laboratories. Cereb Cortex 2000;10:433-42

20. Bernasconi N, Bernasconi A, Caramanos Z, et al. Mesial temporal damage in temporal lobe epilepsy: a volumetric MRI study of the hippocampus, amygdala and parahippocampal region. Brain 2003;126:462-69

21. Loevner LA, Grossman RI, Cohen JA, et al. Microscopic disease in normal- appearing white matter on conventional MR images in patients with multiple sclerosis: assessment with magnetization-transfer measurements. Radiology 1995;196:511-15

22. Tortorella C, Viti B, Bozzali M, et al. A magnetization transfer histogram study of normal-appearing brain tissue in MS. Neurology 2000;54:186-93

23. Mller MJ, Mazanek M, Weibrich C, et al. Distribution characteristics, reproducibility, and precision of region of interest-based hippocampal diffusion tensor imaging measures. AJNR Am J Neuroradiol 2006;27:440-46

24. Yoshiura T, Mihara F, Tanaka A, et al. Novel method to estimate and display cerebral cortical degeneration using diffusion-weighted magnetic resonance imaging. Magn Reson Med 2005;54:455-59

25. Kantarci K, Jack CR, Xu YC, et al. Mild cognitive impairment and Alzheimer disease: regional diffusivity of water. Radiology 2001;219:101-07

26. Shim YS, Yoon B, Shon Y, et al. Difference of the hippocampal and white matter microalterations in MCI patients according to the severity of subcortical vascular changes: neuropsychological correlates of diffusion tensor imaging. Clin Neurol Neurosurg 2008;110:552-61

27. Mller MJ, Greverus D, Weibrich C, et al. Diagnostic utility of hippocampal size and mean diffusivity in amnestic MCI. Neurobiol Aging 2007;28:398-403

28. Del Bigio MR, Bruni JE. Silicone oil-induced hydrocephalus in the rabbit. Childs Nerv Syst 1991;7:79-84

29. Kalus $\mathrm{P}$, Buri C, Slotboom J, et al. Volumetry and diffusion tensor imaging of hippocampal subregions in schizophrenia. Neuroreport 2004;15:867-71

30. Shepherd TM, Ozarslan E, King MA, et al. Structural insights from high-resolution diffusion tensor imaging and tractography of the isolated rat hippocampus. Neuroimage 2006;32:1499-509

31. Klunk WE, Engler H, Nordberg A, et al. Imaging brain amyloid in Alzheimer's disease with Pittsburgh Compound-B. Ann Neurol 2004;55:306-19

32. Small GW, Kepe V, Ercoli LM, et al. PET of brain amyloid and tau in mild cognitive impairment. N Engl J Med 2006;355:2652-63 